Poster Presentation Abstracts

for young people. Whilst a poorly planned transition is associated with increased risk of non- adherence to treatment and decrease of follow up which can lead to serious consequences for the young persons' health and well-being. There is limited empirical evidence in Ireland on how the process is experienced by young people with mental health problems; how it impacts on their health and wellbeing and how the transition process is managed by all stakeholders.

This study proposes a mixed methods longitudinal design to explore the views of young people, their parents and healthcare professionals (Child Psychiatrist, Registrars, Nurses, Psychologist and Social Workers) on the experiences of transition. The data will be obtained using questionnaires and interviews at five different time periods:

(1) in the child mental health services

(2) interviews with participants before transition (in child services)

(3) distribution of questionnaire to young peopleafter transition

(4 \& 5) interviews with participants after transition (adult services) Placing the young persons experience as central to the development of services and ensuring continuity of care are key priorities of National Children's Strategy (2000) and directly impact the quality of service provision.

529

\section{AUDIT ON SWINE FLU VACCINATION UPTAKE IN STAFF WORKING IN PEDIATRIC DEPARTMENT AT IPSWICH GENERAL HOSPITAL}

\author{
M. Kumar, D. Agarwal
}

NHS Suffolk, Ipswich, UK

Background: H1N1 influenza pandemic management was the most challenging scenario faced by health authorities worldwide recently. Department of health (DOH) in UK after doing epidemiological study of first wave of swine flu (April to Sep 2009) gave recommendations for management of predicted Second wave.One of the important recommendations was to prioritize vaccination of frontline health and social care workers. Previous studies have shown that the uptake of seasonal flu vaccine among healthcare workers has consistently been low.
Objective: To evaluate the uptake rate and reasons for non-uptake of HINI Influenza vaccine in frontline healthcare staff working in a busy Pediatric and Neonatal Unit in a Hospital in East Anglia,U.K.

Method: Prospective study conducted over 4 week period during Second Wave of Swine Flu Pandemic. A simple anonymised questionnaire was equally distributed throughout various areas of pediatric and Neonatal department. Staff was randomly informed verbally and actively encouraged to ensure maximum responses.

Results: $46(62 \%)$ out of 74 respondants were not vaccinated. $30(65 \%)$ out of 46 staff were not vaccinated because of Lack of information either relating to vaccine itself (safety, efficacy, side effects); its availability and accessibility .16 (35\%) out of 46 said they were "too busy to get vaccinated". Other reasons stated were - unpleasant reaction to seasonal flu vaccine, needle phobia and people being on annual leave.

Conclusion: Despite the pandemic nature of disease and robust mechanisms in place, Swine flu vaccination uptake continued to be poor. Onsite departmental information and vaccination sessions can be helpful to improve uptake.

\section{0}

PERSISTENCE OF IMMUNE RESPONSE TO CANDIDATE MENINGOCOCCAL SEROGROUPS A,C,W-135,Y TETANUS TOXOID-CONJUGATED VACCINE (MENACWY-TT) UP TO 42 MONTHS FOLLOWING PRIMARY VACCINATION

\section{L. Østergaard ${ }^{1}$, V. Bianco², M. Van der Wielen², J. Miller ${ }^{3}$}

${ }^{1}$ Department of Infectious Diseases, Skejby Sygehus, Aarhus University Hospital, Aarhus, Denmark, ${ }^{2}$ GSK Biologicals, Wavre, Belgium, ${ }^{3}$ GSK Biologicals, King of Prussia, PA, USA

Background and aims: A primary dose of the candidate meningococcal $\mathrm{A}, \mathrm{C}, \mathrm{W}-135, \mathrm{Y}$ tetanus toxoid-conjugated vaccine (MenACWY-TT) was immunogenic and well tolerated in subjects aged 1519years(NCT00126945). Here, the persistenceofthe immune response to MenACWY-TT was compared with the MenA, C,W-135, Y polysaccharide vaccine Mencevax ${ }^{\circledR}$ ACWY (MenPS), and was evaluated up to 42 months after primary vaccination.

Methods: This series of phase II, open, controlled persistence studies included subjects who received 
one dose of MenACWY-TT ( $\mathrm{N}=24)$ or MenPS $(\mathrm{N}=26)$ in study NCT00126945. Persistence of functional antibodies, detected by serum bactericidal activity assay using rabbit serum as exogenous source of complement (rSBA, cut-off 1:8), was determined at 18,30 and 42 months post-vaccination. Serious adverse events (SAEs) were recorded retrospectively up to 42 months post-vaccination.

Results: rSBA titres $\geq 1: 8$ and geometric mean titres (GMTs) for the available timepoints are shown in the Table. All subjects in each group had rSBA titres $\geq 1: 8$ against all serogroups at all timepoints except for the MenPS group against MenC at Month 42. GMTs were higher than pre-vaccination levels at all timepoints post-vaccination in both groups. No SAEs related to vaccination were reported.

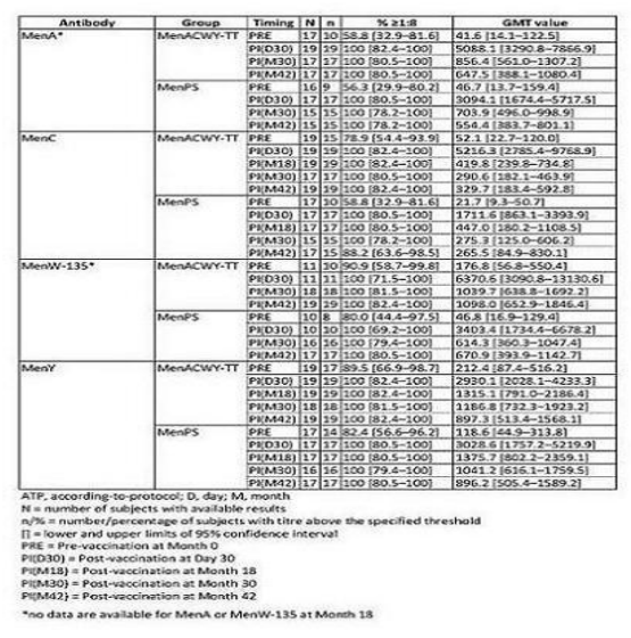

[rSBA $\geq 1: 8$ \& GMTS (ATP cohort for persistence; M42)]

Conclusions: All subjects receiving MenACWYTT retained rSBA titres $\geq 1: 8$ up to 42 months after primary vaccination, indicating that seroprotection following primary vaccination extends more than 3 years.

Mencevax is a trademark of GlaxoSmithKline group of companies.

\section{1}

\section{PARENTS' OPINIONS REGARDING HPV VACCINATION}

D. Konstantelos ${ }^{1}$, T. Syriopoulou ${ }^{1}$, A. Koulouri ${ }^{1}$,

D. Karakaidos ${ }^{2}$, P. Giannakopoulou ${ }^{1}$, S. Psychogiopoulou ${ }^{1}$, N. Karli ${ }^{1}$

${ }^{1}$ Pediatrics, General Hospital of Kalamata, Kalamata, ${ }^{2}$ Pediatrics, General Hospital of Nikea, Piraeus, Greece

Background and aims: To determine opinions regarding vaccination against human papillomavirus (HPV) among parents in Kalamata, Greece.

Methods: During the winter of 2010, 302 parents, with children of female sex 9 to 17 years old, completed a 12-question survey that has been distributed in random local schools evaluating their opinions about vaccines, HPV and the HPV vaccine, and mandatory childhood vaccination programs. Descriptive statistics were calculated for all survey items.

Results: Although $93,7 \%$ of children have been vaccinated according to mandatory vaccination programs in general, the HPV vaccination percent was very low $(1,9 \%)$. From the unvaccinated population $98,1 \%, 54,4 \%$ of parents intended to have their daughters undergo vaccination against HPV in the future. $46,4 \%$ in total thought that HPV vaccination should be mandatory. About the vaccine effectiveness, $35,1 \%$ believed that it gives adequate protection, $28,5 \%$ great protection and only $3,6 \%$ total protection. On the other hand, $21,5 \%$ thought that it offers minimum protection and $3,3 \%$ no protection at all. Their major concerns were that the vaccine is not safe $(40,7 \%)$ and that it had too many adverse effects $(41,7 \%) .79,4 \%$ of the surveyed stated that the information given by the health authorities and the media, regarding HPV vaccination, were insufficient.

Conclusions: Although a large percentage of parents intend to vaccinate their children with HPV vaccine sometime in the future, vaccination rates remain low. Better information given by health authorities as well as more data on safety and efficacy of the vaccine should help improve overall HPV vaccination rates. 